
approches théoriques et méthodologiques

\title{
Connaissance : pseudo-concept partiellement opératoire
}

Knowledge as a Partially Operational Pseudo-Concept

\section{Serge Agostinelli}

\section{CpenEdition}

Journals

Édition électronique

URL : http://journals.openedition.org/edc/3883

DOI : 10.4000/edc.3883

ISSN : 2101-0366

Éditeur

Université Lille-3

Édition imprimée

Date de publication : 1 décembre 2012

Pagination : 65-76

ISBN : 978-2-917562-08-6

ISSN : $1270-6841$

Référence électronique

Serge Agostinelli, «Connaissance : pseudo-concept partiellement opératoire », Études de

communication [En ligne], 39 | 2012, mis en ligne le 16 décembre 2014, consulté le 20 avril 2019. URL http://journals.openedition.org/edc/3883 ; DOI : 10.4000/edc.3883

Ce document a été généré automatiquement le 20 avril 2019.

(c) Tous droits réservés 


\title{
Connaissance : pseudo-concept partiellement opératoire
}

\author{
Knowledge as a Partially Operational Pseudo-Concept
}

\author{
Serge Agostinelli
}

1 L'objectif de cet article est de donner un point de vue sur les raisons des glissements notionnels liés au concept de connaissance. Le peu d'approches conceptuelles du champ de l'organisation et/ou de la gestion des connaissances, nous amène à réfléchir à l'absence de position épistémologique claire sur la façon dont se construit ou est utilisé le concept de connaissance. Dès lors, l'organisation et la gestion d'un objet, lorsqu'on ne sait pas comment ou de quoi il est constitué et éventuellement où en trouver les traces, devient un domaine refuge auquel tous pensent pouvoir se rattacher.

\section{Position du problème}

2 Au-delà d'une faiblesse méthodologique qui amalgame les connaissances aux informations (Jeanneret, 2000) voire aux supports (Naccache, 2010) et d'une approche conceptuelle défaillante qui amalgame savoir, croire et faire (Fontanille, 1987), c'est essentiellement à l'emploi opératoire des connaissances comme des représentations formelles d'un système conceptuel et leurs effets structurants - véritable mythe d'une cognition formelle - qu'il faut attribuer le succès d'un pseudo-concept, sans substance mais partiellement opératoire. En effet, d'un point de vue général, lorsqu'elles ne sont pas disciplinaires, les connaissances s'expriment dans le cadre d'une théorie holiste de l'homme. Les modèles descriptifs sont alors plus proches des théories sociales que d'une épistémologie spécifique. Pourtant le terme de connaissance est bien un concept et la théorie reste à construire avec, comme premier travail, une clarification des termes employés et de leurs relations.

3 Le paradigme cartésien disjoint le sujet et l'objet, avec pour chacun sa sphère propre. Cette dissociation traverse de part en part l'univers (sujet vs objet ; âme vs corps ; esprit vs matière...) avec prédominance de la logique d'une sphère sur l'autre. Cela permet de 
penser la seconde, à partir d'une identification de la structure de l'objet, dans les termes de la première. Ce paradigme détermine donc une double vision du monde : d'une part, un monde d'objets soumis à observations, expérimentations, manipulations; d'autre part, un monde de sujets se posant des problèmes d'existence, de communication, de conscience, de destin... La connaissance désigne alors un rapport de la pensée à une réalité extérieure. Elle est ici une adéquation de l'esprit à l'objet, un mode de mise en relation de phénomènes distincts dont on suppose qu'ils ont des propriétés communes qui donnent un sens à une situation observée. Par exemple, les termes de connaissances communes (Lewis, 1969; Douglas, 1986), connaissances pratiques (Rippere, 1994), connaissances intersubjectives (Nadeau, 1999), connaissances collectives (Berger et Luckman, 1966) sont aussi des constructions symboliques: les idées inadéquates que nous nous faisons des choses; les rapports qui nous composent et composent les choses ; l'essence même des choses qui dépasse les rapports (Deleuze, 1981). L'adjonction d'adjectifs désigne des catégories de regroupement et de classement. On parle alors des connaissances déclaratives sur des informations factuelles, procédurales sur des savoir-faire, conditionnelles sur les conditions de l'action (Mendelshon, 1995). Cette catégorisation devient une représentation formelle d'un rapport au monde, un outil efficace pour la mise en relation de phénomènes à partir d'un ensemble de conditions nécessaires et suffisantes. Nous sommes ici dans un système de propriétés, qu'ont entre elles ou non les connaissances, construit pour l'opérationnalisation, Dès lors, les limites du champ de la gestion des connaissances évoluent en fonction des conditions d'observation des situations dans lesquelles le concept de connaissance est mis en œuvre.

4 Suivant ce point de vue, ce serait l'opérationnalisation du concept sur le terrain qui provoquerait les glissements entre champs d'activités et de recherche voisins mais à visées différentes. La connaissance devient ici un concept-en-acte, ou un concept-en-situation (Vidal-Gomel et Rogalski, 2007), construit en fonction d'une analyse finalisée. La gestion des connaissances est alors liée aux finalités et indépendante de la nature symbolique des connaissances. Liées aux finalités, elles sont alors des ensembles de propriétés pour agir, pour résoudre des problèmes. Par exemple, les connaissances conceptuelles permettent d'analyser, de comprendre, d'agir ; les techniques donnent les méthodes, les procédures ; les humaines se retrouvent dans les relations intra et interpersonnelles... Toutefois, si les finalités nous informent sur les aspects inévitables, obligatoires, contraignants de la gestion des connaissances, elles ne nous disent rien (ou peu) sur ces connaissances qui agissent comme des outils produisant du sens et donc produisant des actions. Le risque est donc d'amalgamer le sens et l'action, ou de les réduire l'un à l'autre. Le problème est bien là; si la pensée fait référence au concept, alors le concept est bien une unité signifiante de la pensée. Il a une fonction intégrante sur l'objet des finalités de l'action qui lui donne une forme systémique et récursive. Ainsi, analyser, comprendre, agir... relèvent bien de la gestion des connaissances car ces activités signifient quelque chose pour nous mais surtout parce qu'elles sont significatives des rapports qui nous lient au terrain ou aux choses observées et à l'essence même des choses qui dépasse les rapports.

5 Les connaissances sont en effet un mode de mise en relation de phénomènes. Elles sont généralisables à des événements distincts et donnent un sens personnel ou collectif à une situation. Le statut des connaissances est donc bien épistémique, social et personnel. L'appréciation de ces trois dimensions explique pourquoi les connaissances ne sont pas seulement des outils applicables au monde extérieur mais bel et bien une construction symbolique. Dans une approche kantienne, construire un concept, c'est présenter 
l'intuition a priori qui lui correspond et sa présentation est un acte consistant à pourvoir un concept d'une intuition correspondante (Freuler, 1992). En tant que concept, les connaissances sont donc autant de questions ouvertes qui conduisent à la recherche d'un type d'instrument pour construire une réalité en fonction des buts et contraintes et par l'intermédiaire des informations perçues sur la situation. Une connaissance correspond ainsi à une manière de poser un problème et d'agir pour le résoudre. Toutefois, le concept de connaissance est-il une notion abstraite constitutive de notre vision du monde ou/et une unité d'analyse pour des champs d'observation? En d'autres termes, est-ce un outil pour l'action ou un instrument pour la pensée? Le problème est donc de savoir si le concept est réductible à l'état cognitif par lequel nous appréhendons les choses ou un objet cognitif distinct de l'action.

\section{Pseudo-concept ?}

6 Dans une logique cartésienne, c'est l'esprit qui est le fondement de notre connaissance du réel : « ... je suis une chose vraie, et vraiment existante; mais quelle chose ? Je l'ai dit: une chose qui pense » (Aimé-Martin, 1838, 69). C'est donc l'homme (le sujet) pensant qui fonde la connaissance et l'esprit n'est pas assujetti au monde. Une connaissance relève alors de la représentation mentale explicite et identifiable à laquelle sont associées les conditions nécessaires et suffisantes de son application. La connaissance des choses ne vient pas de notre expérience sur le monde, de nos sensations; elle appartient à l'esprit. Les expériences nous informent seulement de l'existence des choses. Cette vision cartésienne de la connaissance s'adapte particulièrement bien à une représentation formelle d'un rapport au monde. Le concept de connaissance fait ici référence à quelque chose qui, dans la réalité, est pratique, intersubjectif, collectif et il appartient au concept d'être vérifiable dans la réalité.

7 En revanche dans une logique kantiennne (Kant, 2006), les objets de notre connaissance ne sont que des représentations et la connaissance de la réalité ultime est impossible (Meyer, 2007). Pour connaître, il faut à la fois s'intéresser à l'objet étudié et à l'homme qui l'étudie (Thiebaut, 2008, 63). Ici, la connaissance est une mise en relation entre l'objet et l'intentionnalité liée à son étude. La connaissance des phénomènes résulte donc d'une construction intentionnelle, effectuée par le sujet. Dès lors, le concept avec lequel on donne du sens aux phénomènes n'est pas plus qu'une propriété, une fonction ou une relation que l'on attribue à notre réalité. Il n'est donc pas é tonnant que les auteurs qui s'intéressent aux connaissances se retrouvent sur des champs différents tout en pensant travailler sur la même chose. Cette confusion est encore plus marquée lorsqu'on questionne la connaissance comme un construit ou comme un objet intentionnel (Kalinowski, 1996). Les deux aspects sont bien sûr étroitement liés mais ne sont pas réductibles l'un à l'autre.

Suivant ces remarques, le concept de connaissance ne peut pas être seulement envisagé comme une forme de généralisation extrudée de la liaison concrète et empirique dans lequel il est utilisé. À cette généralisation qui formalise une construction (individuelle ou collective), il convient d'associer une forme d'indexicalité, une contextualisation de l'objet intentionnel. Pour bien montrer cette différence, reprenons l'exemple de Kalinowski $(1996,190)$ :

Ulysse n'est qu'un concept, mais il est conçu à l'instar d'un homme réel. Par conséquent, Ulysse peut être considéré soit comme concept construit, concept 
étant comme tout concept un objet intentionnel, soit comme objet intentionnel conçu à l'instar d'un homme. Il y a donc l'objet intentionnel concept et l'objet intentionnel Ulysse, celui-ci étant identique à celui-là, mais pouvant être envisagé par nous, à la suite d'une distinction de raison sous l'un et sous l'autre aspect. Au total, nous pouvons parler du concept en tant que concept, en l'occurrence construit; du concept en tant qu'objet intentionnel, abstraction faite de sa ressemblance à un homme; et de l'objet intentionnel qu'est ce concept en tant qu'objet ressemblant à un homme.

Pour revenir aux connaissances, prenons par exemple une connaissance procédurale (George, 1988) comme structure de contrôle directement utilisable dans la réalisation de l'action: le savoir-faire, le comment faire. Avec cette forme de connaissance, nous mettons en œuvre des capacités cognitives et motrices fondées sur un système d'association plus ou moins complexes entre des stimuli, des réactions physiques ou intellectuelles. Cependant, selon une réduction logique, beaucoup d'expressions sont privées de leur signification par leur généralisation. Les expressions de connaissances communes, pratiques, intersubjectives, collectives... sont toutes des pseudo-concepts. En effet, si suivant le champ d'observation elles ont leur signification, ces expressions ne peuvent pas être utilisées hors contexte. Elles sont simplement des allusions aux représentations associées et aux sentiments qui n'accordent pas de signification aux expressions. Elles échouent à satisfaire les critères empiriques de richesse du fond et les définitions données sont des pseudo-généralisations. Nous pouvons même penser qu'il s'agit :

d'une réunion, sous forme d'un système complexe, d'une série de généralisation qui, par son apparence extérieure, coïncide parfaitement avec un concept mais qui, par sa nature génétique, par les conditions de son apparition et de son développement, par les liaisons causales dynamiques qui en sont la base, n'est nullement un concept. Extérieurement, c'est un concept, intérieurement, c'est un complexe. C'est pourquoi nous l'appelons pseudo-concept (Vygotski, 1985, 171).

\section{Partiellement opératoire?}

10 Le concept de connaissance est donc un système complexe (cf. Morin, 1990) construit (individuellement ou collectivement) et fondé sur une intentionnalité contextuelle, autrement dit un pseudo-concept. Celui-ci est utilisé pour ses possibilités à contribuer au développement et à la structuration d'un domaine spécifique. L'observation de l'organisation et de la gestion des connaissances relève donc d'une analyse de la complexité dans laquelle se soudent de manière indiscernable, indissociable et indissoluble les idées, les rapports et l'essence. Cette complexité questionne bien sûr les représentations mentales symboliques mais également les caractères individuels vs collectifs, experts $v s$ novices, tacites vs explicites... Autrement dit, à cette complexité il convient d'appliquer un groupement à partir des caractéristiques observables au terme duquel sont associées des actions, des pratiques observables ou de pensées inférables appartenant à un même champ de recherche (l'enseignement, l'informatique, la sociologie, etc.). Quand ces groupements sont effectués, le caractère opératoire du pseudo-concept justifie les propriétés de stabilité, de réversibilité, de déduction de son utilisabilité concrète et logique. Ce caractère est particulièrement marqué lorsque la recherche est confrontée au problème d'organiser de manière cohérente les activités dans l'espace et dans le temps de la discipline qui la reconnait. La structure opératoire est alors confrontée à deux défis : comment structurer un domaine de recherche qui respecte 
l'ensemble des contraintes du terrain tout en se dégageant le plus possible des constructions symboliques sous-jacentes? Comment construire un domaine de recherche de manière compositionnelle compte tenu des interactions qui existent entre l'action et la pensée, entre un concept qui organise notre pensée mais qui est aussi le produit de celleci?

11 Les réponses se trouvent dans une logique d'action (Bernoux et al., 2005) avec laquelle on cherche à prendre en compte la complexité du pseudo-concept sous toutes ses formes possibles. Les domaines d'actions (organisation et gestion) des connaissances sont donc cadrés, structurés afin de permettre les compréhensions plus ou moins partiellement déterminées par le concept et les pratiques. Cette logique opératoire articule les acteurs (chercheurs, discipline, terrain...) à la fois stratégiquement, scientifiquement et culturellement. Elle autorise les objectifs stratégiques généraux, les orientations d'action et les moyens d'opérationnalisation privilégiés par chacun des domaines de recherche. Le domaine devient alors une construction symbolique, un système de valeurs et de normes qui donne aux chercheurs le sens d'une identité commune (Cohen, 1985) au sein d'une communauté scientifique.

\section{Absence d'une position épistémologique}

12 À l'intérieur de cette communauté, les relations existant entre les champs d'activités et les recherches sont voisines mais ont des visées différentes. La complexité ne favorise pas l'homogénéité et il est plus simple de dire sur quel terrain on travaille que d'avancer les concepts avec lesquels on travaille. De plus, revendiquer une appartenance à un domaine, c'est aussi reconnaître que la discipline ne recouvre pas la complexité d'un terrain. Ceci est d'autant plus significatif lorsque le concept majeur du domaine peut être envisagé par plusieurs paradigmes (Kuhn, 1972) tout en restant opérationnel sur chacun des différents aspects. Les raisons des glissements notionnels liés au concept de connaissance trouvent donc leur source dans la difficulté à savoir si l'on travaille avec un concept ou un pseudoconcept, si l'on privilégie l'abstraction ou l'opératoire. Dès lors, il reste à savoir quel positionnement épistémologique favorise l'une ou l'autre approche du concept de connaissance. Suivant le paradigme choisi, nous nous trouvons donc avec deux modèles différents pour décrire des connaissances. Ces derniers coexistent aujourd'hui et envisagent les connaissances dans deux approches pratiques: l'une sémiotique, l'autre sémantique.

\section{Approche cartésienne}

13 Le paradigme cartésien s'érige en obstacle à la compréhension du concept de connaissance. C'est la vision rationnelle qui va du simple au complexe et prône que toute connaissance est susceptible d'être expliquée plutôt que décrite. Cette explication se fonde alors sur l'identification de la structure des connaissances, dont il est certain qu'elle existe et qu'elle est unique et invariante. L'observation des faits et l'expérience de pensée sont le fondement de la connaissance. La connaissance ne vient pas du corps, des sensations, mais de l'esprit. Les sens nous informent seulement de l'existence de l'objet (Descartes, 1724). C'est ce paradigme qui détermine la double vision du monde et donne donc naissance au problème. Toutefois, ce problème est relatif. En effet, il ne remet pas en question les connaissances puisqu'elles partent de faits observables, des observations qui 
consistent toujours à mettre en relation un objet et un observateur. Toutefois, on est ici dans le mythe d'un univers de connaissances que l'on peut circonscrire ou représenter. D'ailleurs, pour chaque phénomène observé, il existe une base observationnelle complètement indépendante de toute théorie et l'observateur a la possibilité de réduire toutes les données théoriques à des données observables. Ici, la construction de connaissances constitue une procédure de traitement des données par laquelle les résultats $\mathrm{du}$ traitement génèrent des entrées pour d'autres traitements. Les connaissances sont stockées sous la forme de blocs qui constituent la base de connaissances disponibles. Ces blocs sont reliés par des réseaux sémantiques. Ces réseaux de blocs structurent les connaissances en schémas prototypiques utilisables dans plusieurs situations et ces schémas sont instanciés par les informations de la situation particulière. C'est un modèle opératoire des connaissances à la fois prototypique et procédural (relation entre action et but).

\section{Le modèle sémantique}

14 Le modèle sémantique cognitif est largement utilisé aujourd'hui par les sciences formelles. Ce modèle est constitué d'un ensemble de nœuds étiquetés (représentant généralement des connaissances), d'un ensemble de liens orientés et étiquetés entre ces nœuds (représentant généralement des relations entre des connaissances) et d'un ensemble d'opérations constituant les mécanismes de mise en relation des connaissances. Ceci forme le réseau sémantique qui hiérarchise des structures cognitives, c'est-à-dire l'organisation mentale des connaissances (Houdé, 2004). Cette hiérarchisation correspond à l'hypothèse d'économie cognitive dans laquelle une information qui autorise un ensemble de connaissances n'apparaît qu'une fois, par exemple " vole » pour les oiseaux. Le verbe « voler » devient typique de la catégorie oiseau. Bien sûr, cette information (ici, " un oiseau vole») peut être ou ne pas être essentielle mais laisse penser que les connaissances sont représentées par une liste de caractéristiques aux propriétés perceptives plus ou moins en corrélation avec un prototype catégoriel. Les connaissances sont définies par une ou des propriétés qu'ont, ou n'ont pas, entre elles les informations. Elles sont des représentations formelles d'un système conceptuel fondé sur un modèle sémantique ontologique (Raimbault et al., 2008).

\section{Approche kantienne}

15 En revanche, avec l'approche constructiviste, les connaissances n'existent pas par ellesmêmes, elles sont le résultat des constructions mentales. Elles sont des constructions subjectives. La connaissance est incluse dans une ontologie. C'est un instrument intellectuel consensuel, qui n'est pas propre à un individu, mais partagé par un groupe et sa construction est constitutive du système personnel de représentation du monde. La construction de connaissances constitue un système de mise en relation des individus, des informations, une situation et un contexte. Les connaissances sont composées d'une collection de données séparées qui constituent un système périphérique de traitement de l'information. Le traitement informationnel participe à la construction des connaissances qui est de nature cognitive et directement issue des interactions. C'est un modèle consensuel des connaissances à la fois fonctionnel et contextuel. 


\section{Le modèle sémiotique}

Avec le modèle sémiotique, les connaissances sont définies par une construction de sens à partir des types de signes (les informations) et de leur relation avec les objets (les données). Elles sont le résultat d'une médiation entre le cognitif et le réel, d'une médiation sémiotique (Vygotski, 1985). À travers cette médiation, les connaissances se construisent dans une négociation (intra et inter personnelle) co-contextualisée visant à « donner du sens » aux nouvelles informations que l'individu doit organiser. Le type de médiation dépend du niveau d'abstraction, d'apprentissage et du contexte culturel. Les connaissances relèvent ici d'un processus de mise en relation : a) d'un fait qui est traduit ou devient une donnée grâce à une perception (observer un réel), b) d'une donnée qui est traduite en information grâce à une compétence (construction du sens), c) d'une information qui devient une connaissance grâce à une capacité (structuration fonctionnelle) et e) d'une connaissance qui devient un savoir grâce à une performance (action). L'information est ici un instrument qui permet de prolonger et d'adapter la connaissance pour obtenir une meilleure perception. La construction personnelle du sens est alors mise en avant avec le caractère intentionnel et finalisé des activités humaines.

\section{Conclusion}

Loin d'être dichotomique, la façon dont on représente des connaissances n'appartient pas à un courant ou un autre et la formation d'un concept relève d'un continuum à mettre en relation avec la nature des modèles que nous venons de décrire brièvement.

Toutefois, la façon dont on pense que les connaissances se construisent induit un choix dans les modèles d'organisation et de gestion. En ce sens, et en faisant de l'organisation des connaissances, la formalisation d'un réel possible compatible avec ces apparences, celle-ci se réduit toujours à un modèle. Aussi, les connaissances ne peuvent être dissociées de la pluralité des modèles et par conséquent, de leur contingence.

BIBLIOGRAPHIE

Aimé-Martin L., (1838), Oeuvres philosophiques, Paris, Auguste Desrez.

Bernoux P., Amblard H., Herreros G. et Livian Y.-F., (2005), Les nouvelles approches sociologiques des organisations, Paris, Seuil.

Cohen A., (1985), The symbolic construction of community, London, Routledge.

Descartes R., (1724), Les méditations métaphysiques, Paris, Robert - Marc d'Espilly.

Deleuze G., (1981), Les cours de Gilles Deleuze : http// :www.webdeleuze.com/, date de la dernière visite : 1 février 2012.

Douglas M., (1986), Risk Acceptability According to the Social Sciences, London, Routledge. 
Fontanille J., (1987), Le savoir partagé, Amsterdam, Hadès, John Benjamins Publishing.

Freuler L., (1992), Kant et la métaphysique spéculative, Paris, Vrin, Bibliothèque des Textes Philosophiques.

George C., (1988), « Interactions entre les connaissances déclaratives et procédurales », in P. Perruchet (éd.), Les automatismes cognitifs, Liège, Pierre Margada, pp. 103-137.

Houdé O., (2004), Vocabulaire de sciences, New-York, Routledge.

Jeanneret Y., (2000), Y a-t-il (vraiment) des technologies de l'information ?, Lille, Presses universitaires-Septentrion.

Kalinowski G., (1996), Sémiotique et philosophie, à partir et à l'encontre de Husserl et de Carnap, Limoges, Presses universitaires de Limoges.

Kant E., (2006), Critique de la raison pure, Paris, PUF.

Kuhn T., (1972), La structure des révolutions scientifiques, Paris, Flammarion.

Lewis D. K., (2002), Convention : A philosophical study, Cambridge, Mass., Harvard University Press.

Luckmann T. et Berger P., (2006), La construction sociale de la réalité, Paris, Armand Colin.

Mendelsohn P., (1995), « Peut-on vraiment opposer Savoirs et Savoir-faire quand on parle d'apprentissage ? », in Les Entretiens Nathan, Actes V, Savoirs et Savoir-faire, pp. 21-40.

Meyer C., (2007), Une histoire des représentations mentales, contribution à une archéologie de la société de la connaissance, Paris, L'Harmattan.

Morin E., (1990), Introduction à la pensée complexe, Paris, Éditions Sociales Françaises.

Naccache L., (2010), Perdons-nous connaissance? De la Mythologie à la Neurologie, Paris, Odile Jacob.

Nadeau R., (2006), « Objectivité », in D. Lecourt (éd.), Dictionnaire d'histoire et philosophie des sciences, Paris, PUF, pp. 698-706.

Raimbault T., Briand H., Genest D., Lehn R. et Loiseau S., (2008), « Une synthèse des modèles de représentation des connaissances à base de Graphes Conceptuels et OWL », Revue des nouvelles technologies de l'information, oㅡㄴ 12, pp. 45-66.

Rippere V., (1994), « An Empirical Anthropological Method for Investigating Common Sense », in J. Siegfried (éd.), The Status of Common Sense in Psychology, Norwood, N.J., Ablex, pp. 247-258.

Thiébaut C., (2008), Dessine-moi un avenir professionnel : nos représentations de l'orientation professionnelle, Paris, L'Harmattan.

Vidal-Gomel C. et Rogalski J., (2007), La conceptualisation et la place des concepts pragmatiques dans l'activité professionnelle et le développement des compétences, @ctivités, vol. 4, $\mathrm{n}^{\circ} 1$, pp. 49-82.

Vygotski L., (1985), Pensée et langage, Paris, Messidor/Éditions Sociales.

\section{RÉSUMÉS}

Cet article donne un point de vue sur les raisons des glissements notionnels liés au concept de connaissance. Le concept de connaissance change suivant la position épistémologique du chercheur. En fonction d'une approche cartésienne ou kantienne, le concept est un objet qui relève de l'esprit ou une relation entre l'objet et l'intentionnalité liée à son étude. Dès lors, la 
connaissance peut être une notion abstraite constitutive de notre vision du monde ou une unité d'analyse pour des champs d'observation. Du concept, on passe au pseudo-concept qui relève d'un système complexe dans lequel la logique d'action lui donne son caractère opératoire.

This paper provides a perspective on the causes of the conceptual shift related to the notion of knowledge. The concept of knowledge changes according to the researcher's epistemological position. According to the Cartesian or the Kantian approach, the concept is either an object within the mind or a relationship between the object and the intentionality related to its study. Therefore, knowledge can be an abstraction constitutive of our vision of the world or a unit of analysis for the fields of observation. From this concept, we move to the pseudo-concept, part of a complex system in which the logic of action gives it its operational character.

\section{INDEX}

Mots-clés : connaissance, concept, épistémologie, paradigme, sémantique, sémiotique

Keywords : knowledge, concept, epistemology, paradigm, semantic, semiotic

\section{AUTEUR}

\section{SERGE AGOSTINELLI}

Serge Agostinelli, UMR CNRS 7296 - Laboratoire des Sciences de l'Information et des Systèmes, Université d'Aix-Marseille 